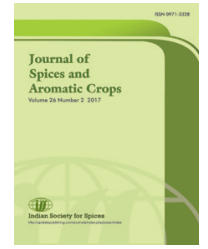

\title{
Essential oil content of cardamom (Elettaria cardamomum Maton) by hand-held electronic nose
}

N K Leela*, A M Muneeb, S Mukherjee ${ }^{1}$, D Ghosh ${ }^{1} \&$ N Bhattacharya ${ }^{1}$

ICAR-Indian Institute of Spices Research, Kozhikode- 673 012, Kerala.

*E-mail: leela@spices.res.in

Received 14 February 2017; Revised 28 June 2017; Accepted 23 October 2017

\begin{abstract}
Cardamom (Elettaria cardamomum Maton.) is one of the most expensive spices in the world. Essential oil is the most functionally important component of cardamom and is described as sweet, spicy, warm, camphoraceous and citrusy. The quality of cardamom was assessed by the essential oil content and its composition. Development of hand-held E-nose to evaluate quality of cardamom in terms of its essential oil content and its validation are reported.
\end{abstract}

Keywords: cardamom, hand-held electronic nose, 1,8-cineole, á-terpinyl acetate.

Cardamom (Elettaria cardamomum Maton.) a member of Zingiberaceae family is a herbaceous perennial, and is one of the most expensive spices in the world. Its dried fruits are used as spice and flavoring agent in processed foods, tonics, liquors and perfumes. The quality of cardamom is governed by its volatile oil constituents. More than thirty constituents that contribute to aroma of the oil have been characterized by GC-FID and GC-MS analysis (Chempakam \& Sindhu 2008). These methods are time consuming and need specialized expertise and hence the use of non-destructive techniques such as the artificial olfactory system (AOS) is being explored for fast and objective measurement of quality. Present study is an attempt to determine the essential oil content of cardamom by the newly designed hand-held E-nose (HEN).
E-Nose consists of an assemblage of metal oxide semiconductor (MOS) sensors, an odour delivery system, PC-based data acquisition system and a pattern recognition system. Enose provides fast, automated as well as real time classification and detection of quality and can be easily trained to suit different applications to detect a variety of odours. In agriculture, E-nose is used for testing the quality of tea, level of maturity of apple and pears, shelf-life of mango chips, apple, fresh cut vegetables and grape wine and freshness of milk, dairy products, meat, fish, egg etc. (Berna 2010). The use of hand-held E-nose for determination of quality of tea, cumin and pepper has been demonstrated (Bhattacharya et al. 2008, Mamatha et al. 2008, Renata et al. 2010). 
At trader's level quality of cardamom is generally evaluated based on the external appearance, colour and size. The assessment of the oil content in capsules will certainly add value to the produce. As per available information there is no report on the quality evaluation of cardamom using E-Nose. Hence, the present work was attempted to determine the quality of cardamom in terms of its essential oil content using hand-held electronic nose (HEN).

Selection of sensors: Preliminary selection of sensors was conducted at C-DAC, Kolkata using an Electonic Nose and Vision (ENV) System. Three different sensor arrays were used for the analysis with a total of twenty one commercially available MOS sensors (Figaro Engineering Japan). The sensor array-1 consisted of the sensors TGS 832, TGS 826, TGS 825, TGS 2620, TGS 2444, TGS 2602 and TGS 2611. Sensor arrays -2 and 3 consisted of TGS 822, TGS 2611, TGS 2442, TGS 880, TGS 2610, TGS 2612 and TGS 832, TGS 823, TGS 2620, TGS 816, TGS 830 , TGS 2611 ,TGS 2620 and TGS 2600 respectively. Response from these sensors using various combinations of $\alpha$ terpinyl acetate and 1,8-cineole (namely 100\%, $75 \%, 50 \%$ and $25 \%$ ) were recorded (Table 1 ) and individual responses were subjected to principal component analysis (PCA). PCA loadings of sensors are widely used to analyze the sensor scores and to select suitable sensors (Gardner 1991; Abdullah et al. 2012). The sensor TGS 823 showed highest score where as TGS 816 showed no response for the combinations tested. The sensors having high response and more sensitivity to the combinations of á-terpinyl acetate and 1,8-cineole were selected, which included TGS 823, TGS 822, TGS 826, TGS 825, TGS 832, TGS 880, TGS 2620 and TGS 2602 (Muneeb et al. 2014). Further, the hand-held electronic nose E-Nose (HEN), which is a miniaturized version of the Electronic Nose was modified by updating the sensor array and algorithm. HEN consisted of a sample holder, an odour delivery module, sensor arrays and PCBs (printed circuit boards) for signal conditioning and software. The software options included, user-specific training, testing of unknown sample, report generation, data archival and system diagnosis. The development platform included a firmware in C- language and touch screen based graphic user interface (GUI) with a 16-bit controller microchip hardware. The architecture of the HEN is indicated in Fig.1.

Determination of essential oil content by hydrodistillation method: Cardamom samples collected from ICAR-Indian Institute of Spices Research, Regional Station, Appangala and Indian Cardamom Research Institute (ICRI), Myladumpara were analyzed for seed weight, and essential oil content. Essential oil was extracted from dried capsules ( $25 \mathrm{~g}$ ) by hydro distillation in a Clevenger- type apparatus for three hours (ASTA 2010). The essential oil separated was collected, made moisture free using anhydrous sodium sulphate and the content was expressed in percentage. Essential oil content of fifty cardamom samples collected from Appangala and Idukki was determined

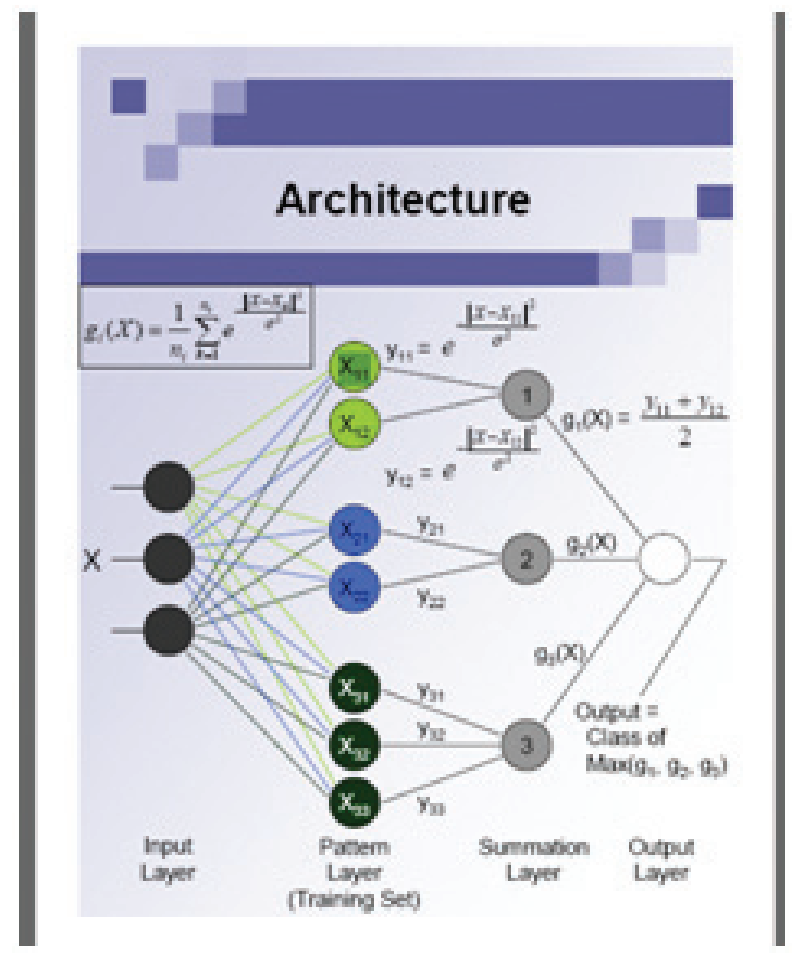

Fig. 1. Architecture of HEN 
Table 1. The PCA loading of individual sensors

\begin{tabular}{|c|c|c|c|c|c|}
\hline \multirow[b]{2}{*}{ Sensor } & \multicolumn{4}{|c|}{ (from $100 \%$ to $25 \%$ ) } & \multirow[b]{2}{*}{$\begin{array}{c}\alpha \text {-Terpinyl } \\
\text { acetate } 100 \%\end{array}$} \\
\hline & Cineole 100\% & ${ }^{*}$ Cineole $75 \%$ & ${ }^{*}$ Cineole $50 \%$ & ${ }^{*}$ Cineole 25\% & \\
\hline TGS-823 & 0.6168 & 0.5769 & 0.6632 & 0.6064 & 0.6881 \\
\hline TGS-826 & 0.4121 & 0.4478 & 0.2932 & 0.4237 & 0.4295 \\
\hline TGS-822 & 0.2698 & 0.2616 & 0.2496 & 0.277 & 0.1788 \\
\hline TGS-832 & 0.2666 & 0.3639 & 0.4053 & 0.3848 & 0.1837 \\
\hline TGS-832 & 0.2634 & 0.2464 & 0.1483 & 0.2359 & 0.1214 \\
\hline TGS-2611 & 0.1494 & 0.1233 & 0.1369 & 0.0969 & 0.3638 \\
\hline TGS-825 & 0.1385 & 0.1789 & 0.1993 & 0.1902 & 0.135 \\
\hline TGS-2611 & 0.0847 & 0.0705 & 0.0722 & 0.0493 & 0.051 \\
\hline TGS-880 & 0.0823 & 0.0881 & 0.0776 & 0.0646 & 0.0467 \\
\hline TGS-2620 & 0.0747 & 0.0814 & 0.0338 & 0.0731 & 0.0761 \\
\hline TGS-2612 & 0.0696 & 0.0606 & 0.0628 & 0.0389 & 0.0408 \\
\hline TGS-2602 & 0.0543 & 0.0645 & 0.0748 & 0.0676 & 0.0289 \\
\hline TGS-2442 & 0.0234 & 0.0264 & 0.0256 & 0.0163 & 0.0149 \\
\hline TGS-2620 & 0.0206 & 0.0225 & 0.0254 & 0.0225 & 0.0263 \\
\hline TGS-2600 & 0.0136 & 0.0113 & 0.0071 & 0.0101 & 0.0081 \\
\hline TGS-2610 & 0.0129 & 0.0152 & 0.0157 & 0.0096 & 0.0103 \\
\hline TGS-2611 & 0.0070 & 0.0021 & 0.0104 & 0.0068 & 0.0052 \\
\hline TGS-2444 & 0.0040 & -0.0041 & 0.0023 & 0.0007 & 0.0005 \\
\hline TGS-2610 & 0.0002 & -0.0003 & 0.0148 & 0 & 0.0004 \\
\hline TGS-830 & 0.0001 & 0 & 0.0001 & 0 & 0 \\
\hline TGS-816 & 0 & 0 & 0 & 0 & 0 \\
\hline
\end{tabular}

${ }^{*}$ Rest-Terpinyl acetate

by hydrodistillation method (ASTA 2010) and used for training HEN.

\section{Training HEN for evaluation of cardamom aroma}

For training HEN, crushed decorticated seeds of fifty cardamom samples (5 g) with known essential oil content (3-7\%) were placed in the sample holder and 'Training method' was selected in the software and sniffing cycle was applied. Once it was completed oil content of the sample as determined by hydrodistillation method was manually entered and saved. Each sample was replicated five times yielding a data set of 250 samples and the training data were generated using the algorithm, probabilistic neural network (PNN). The total data set was divided in 60:40 ratio for training and testing. Forty percent data were tested using $60 \%$ training data with minimum standard deviation $(\sigma)$.

Determination of essential oil content by HEN: For testing samples, the decorticated seeds (5 g) were placed in sample holder of HEN and the 'Testing' option was selected in the software and sniffing cycle was applied. The sniffing cycle consisted of headspace generation (30 s), sampling (50 s), and purging (300 s). During each cycle ten data corresponding to the change in resistance of sensor were generated for each sensor per second. Based on the response of sensors the oil yield with optimized $\sigma$ was calculated by the software and by pressing the option 'Results' the percentage oil yield was displayed as output.

The essential oil yield of the cardamom samples determined by HEN and that by hydrodistillation method are indicated in Table 2. The oil content of capsules varied from 3.8\% to $7.0 \%$ on dry weight basis by HEN analysis and from $3.6 \%-7.4 \%$ by hydrodistillation method. The difference in percent oil yield of the samples by HEN and hydro distillation 
Table 2. Essential oil yield by HEN and hydro distillation method

\begin{tabular}{|c|c|c|c|c|c|}
\hline \multirow[t]{2}{*}{ Normaroma index } & \multicolumn{2}{|c|}{ HEN analysis } & \multicolumn{2}{|c|}{ Hydro distillation method } & \multirow{2}{*}{$\begin{array}{c}\text { Difference in } \\
\text { Oil yield \% (B-A) }\end{array}$} \\
\hline & Oil yield \% (A) & Score & Oil yield \% (B) & Score & \\
\hline 59.19 & 6.7 & High & 7.2 & High & +0.5 \\
\hline 56.52 & 6.3 & High & 6.2 & High & -0.1 \\
\hline 46.96 & 4.8 & Medium & 5.0 & Medium & +0.2 \\
\hline 40.45 & 3.8 & Low & 3.6 & Low & -0.2 \\
\hline 45.11 & 4.0 & Low & 3.6 & Low & -0.4 \\
\hline 59.00 & 6.7 & High & 7.4 & High & +0.7 \\
\hline 47.03 & 5.2 & Medium & 6.0 & Medium & +0.8 \\
\hline 63.94 & 7.0 & High & 6.6 & High & -0.4 \\
\hline 71.61 & 5.9 & Medium & 5.8 & Medium & -0.1 \\
\hline
\end{tabular}

Scale: $<4 \%=$ low; $4.0-6.0 \%=$ medium; $>6.0 \%=$ high (on capsule weight basis)

method was $-0.1 \%$ to $+0.8 \%$. Using HEN the cardamom samples can be classified into low $(<4 \%)$, medium $(4-6 \%)$ and high $(>6 \%)$ based on the oil content; Table 2 shows that the scores obtained by both methods are the same. This is the first attempt to determine oil yield of cardamom by electronic nose. The analysis by HEN is a nondestructive fast method which requires only 5 minutes where as 3 hours are needed for completion of hydro distillation. HEN analysis requires $5 \mathrm{~g}$ of sample where as it is $20-25 \mathrm{~g}$ for hydro distillation. More over HEN is user friendly and can be easily operated. The accuracy and precision of the method can be further improved by training the system with more number of samples.

\section{Acknowledgements}

The authors are thankful to the Director, ICARIISR, Kozhikode for providing facilities and Executive Director, C-DAC, Kolkata and Department of Electronics and Information technology (DietY), Government of India, for financial assistance.

\section{References}

ASTA (2010) Official Analytical Methods of American Spice Trade Association, $4^{\text {th }}$ Edition, Newyork.
Abdullah A H, Adom A H, Shakaff A Y M, Ahmad M N, Zakaria A, Saad F S A \& Kamarudin L M (2012) Hand-held electronic nose sensor selection system for basal stamp rot (BSR) disease detection. In: Third International Conference on Intelligent systems, modelling and simulation (ISMS), 8-15 Feb 2012, IEEE, p.737-742.

Bhattacharyya N, Bandyopadhyay R, Bhuyan M, Bipan T, Ghosh D \& Jana A 2008 Black tea classification and correlation of measurements with tea taster marks. IEEE Trans. Instrum. Measur. 57: 1313-1321.

Berna A Z 2010 Metal oxide sensors for Electronic noses and their application to food analysis. Sensors 10: 3882-3910.

Chempakam B \& Sindhu S 2008 In: Parthasarathy V A, Chempakam B \& Zachariah T J (Eds.), Chemistry of spices, pp.41-58.

Gardner J W 1991 Detection of vapours and odours from a multi sensor array using pattern recognition Part 1-Principal component analysis, Sens. Actuators B: Chemical 4: 109-116.

Mamatha B S, Prakash M, Nagarajan S \& Bhat K K (2008) Evaluation of the flavor quality of pepper (Piper nigrum L.) cultivars by GC-MS, electronic nose and sensory analysis techniques. J. Sens. Stud. 23: 498-513. 
Muneeb A M, Mukherjee S, Ghosh D, Bhattacharya N \& Leela N K 2014 Selection of suitable metal oxide semiconductor sensors to determine essential oil composition of cardamom. In: Dinesh R, Santhosh J Eapen, Senthil Kumar C M, Ramakrishnan Nair R, Devasahayam $S$, John Zachariah $T$ \& Anandaraj M (Eds.), Abtracts - International Symposium on Plantation Crops (PLACROSYM XXI): Converging technologies for sustainability, ICARIndian Institute of Spices Research,
Kozhikode, Kerala, India, 10-12 December 2014, p.200.

Ramon Aparicio, Silvia M Rocha, Ivonne Delgadillo \& María Teresa Morales 2000 Detection of rancid defect in virgin olive oil by the electronic nose. J. Agri. Food Chem. 48: 853-860.

Renata Z W, Sylwia M S, Erwin W \& Mariusz P 2010 Gas chromatography, sensory analysis and electronic nose in the evaluation of black cumin (Nigella sativa L.) aroma quality. Herba Polonica 56: 20-30. 